\title{
APPLICATION OF AN OPTIMAL CONTROL ALGORITHM FOR A GYROSCOPE SYSTEM OF A HOMING AIR-TO-AIR MISSILE
}

\author{
Izabela KRZYSZTOFIK (D)*, Zbigniew KORUBA (D) \\ Department of Applied Computer Science and Armament Engineering, Faculty of Mechatronics and Mechanical \\ Engineering, Kielce University of Technology, Kielce, Poland
}

Received 21 March 2020; accepted 16 October 2020

\begin{abstract}
Missile homing precision depends mainly on the correct determination of the current angle between the Gyroscope System Axis (GSA) and the target line-of-sight (LOS). A gyroscope automatic control system shall ensure spontaneous levelling of this angle, hence, constant homing of the gyroscope system axis in on the LOS, i.e. tracking the target by the head. The available literature on the subject lacks a description of how to use the controlled gyro system in the process of guiding the missile onto the target. In this paper, the authors present the original development of an optimal control algorithm for a gyro system with a square quality indicator in conditions of interference and kinematic influence of the missile deck. A comparative analysis of the $\mathrm{LQR}$ with the $\mathrm{PD}$ regulator was made. $\mathrm{PD}$ regulator parameters are also selected optimally, using the Golubencev method, so that the transition process of the homing system fades over a minimal time, while simultaneously ensuring the overlapping of the gyroscope axis with the target line-of-sight. The computer simulation results have been obtained in a Matlab-Simulink environment and are presented in a graphic form.
\end{abstract}

Keywords: non-linear dynamics, gyroscope system, optimal regulator, guidance, missile flight.

\section{Introduction}

One of the most important air-air missile elements is the homing head. Homing heads utilizing infrared radiation are most commonly used. The head intercepts and tracks an air target. Missile homing involves, primarily, determining an engaged target line-of-sight - a straight line from the homing head to the target (Balakrishnan et al., 2013; Zarchan, 2012).

An optical target coordinator is the basic element of the head. A coordinator optical system is embedded in the gyroscope disc, suspended on the Cardan joint. The inflight task of a target coordinator is to determine the angle between the target line-of-sight (LOS) and the coordinator axis (gyroscope system axis-GSA) or its components or the angular velocities. This operation is automatic, so that the GS control system constantly directs the optical axis onto a moving target. When the gyroscope axis overlaps the target line-of-sight, the missile is deemed to track and follow the target. The sensors measure the angle between LOS and the missile axis and forward it to the autopilot. The autopilot, autonomously uses its own instrumentation to measure the angular position of the target axis relative to the Earth, and then determines control signals and forwards them to the control actuation system (Gapiński et al., 2018; Grzyb \& Stefański, 2016).

Therefore, the accuracy of determining the actual angle between the GS axis and the LOS significantly impacts the precision of an air-air missile homing onto a manoeuvring air target, hence, it increases the efficiency of reaching and destroying the target (Gapinski \& Krzysztofik, 2014). A gyroscope system is subject to the actions of the missile deck, which prevent long-term maintenance of a desired optical axis position with a specified accuracy. Thus, the control system parameters must be selected in an optimal manner so as to minimize the mean squared error and GS dynamic effects appearing within the transition process (Koruba \& Krzysztofik, 2013; Krzysztofik et al., 2017).

Numerous researchers have studied the dynamics and control of a gyroscope system wherein a gyroscope was considered as a symmetrical solid body located on a vibrating support. The spatial position of a gyroscope was described using Euler angles, and the base vibrations adopted in the form of harmonics. Chaotic behaviours within the gyroscope system were mainly studied (Chen \& Ge, 2005; Ge \& Lee, 2005; Lei et al., 2005). Various algorithms for controlling and synchronizing these behaviours were also developed. Diverse control methods, such as

${ }^{*}$ Corresponding author. E-mail: pssik@tu.kielce.pl 
fuzzy logic control (Sargolzaei et al., 2013), sliding mode control (Wang \& Yau, 2011), fuzzy sliding mode control (Chen et al., 2013; Yau, 2008) and adaptive fuzzy sliding mode control (Roopaei et al., 2010) were also considered.

In contrast to the aforementioned papers, this article is a study of the practical application of a gyroscope system suspended on a Cardan joint, as a homing head propulsion element. A system of such type is presented by Gapiński and Stefański (2014), Krzysztofik et al. (2017), Polo et al. (2008). Furthermore, Polo et al. (2008) developed a PID regulator and a procedure for determining its permissible parameters, while Krzysztofik et al. (2017) applied a fuzzy regulator, whereas Gapiński and Stefański (2014) implemented a control algorithm using phase trajectories of control deviations.

This article discusses the most generic GS dynamics model. This is due to the fact that it has taken into account external forces, frame inertia and, most of all, the GS centre of gravity displacement relative to the rotation centre (a so-called "heavy" gyroscope). In addition, the paper concentrates on the control system for an axis of a gyroscope with a missile being its only support, which acts on the gyroscope bearings. Preliminary test reports involving the functioning of an LQR regulator onboard a missile were presented at the DSTA 2019 international conference.

The study involved developing an algorithm for the optimal control of a gyroscope system with a square quality indicator (minimum mean square error of GSA deflection from the target LOS) in conditions of interference in the form of the kinematic action of the missile deck. The gain matrices for an optimal regulator executing the developed algorithm were derived from the algebraic Riccati equation (after prior linearization of non-linear dynamic equations for the GS and expressing form in the form of equations of state). A block diagram of a gyroscope system control system operating within the homing process is shown in Figure 1.

The diagram above shows four main blocks - the homing air-to-air missile, autopilot, controlled gyroscope system and air target. A gyroscope system used within a homing head is a highly non-linear system. Therefore, let us first linearize the GS dynamics model and determine the state and control matrices. Next, the LQR method (equations 19) is used to determine the gain matrix, which is then sent to the input of the optimal regulator. The second regulator input signal is comprised of control deviations based on the current positions of the LOS and GSA. Ultimately, the determined optimum controls are sent to the input of a non-linear gyroscope system.

\section{Governing equations and design of gyro system optimal control algorithm}

A general view of a gyroscope system of a missile homing head, along with adopted coordinate systems is shown in Figure 2. Individual values mean: $O_{m} x y z$ - coordinate system associated with the missile body; $\vec{p}_{m}, \vec{q}_{m}, \vec{r}_{m}-$ components of a missile angular velocity vector; $\vec{V}_{m}-$ missile flight speed vector; $O_{g} x_{1} y_{1} z_{1}$ - coordinate system associated with the outer frame of a gyroscope system; $\mathrm{O}_{g} x_{2} y_{2} z_{2}$ - coordinate system associated with the inner frame of a gyroscope system; $\mathrm{O}_{g} x_{3} y_{3} z_{3}$ - coordinate system associated with the rotor of a gyroscope system; $\theta_{g}, \psi_{g}, \Phi_{g},-$ angles of inner frame rotation, outer frame rotation and specific rotation, respectively; $\vec{U}_{b}, \vec{U}_{c}-$ vec-

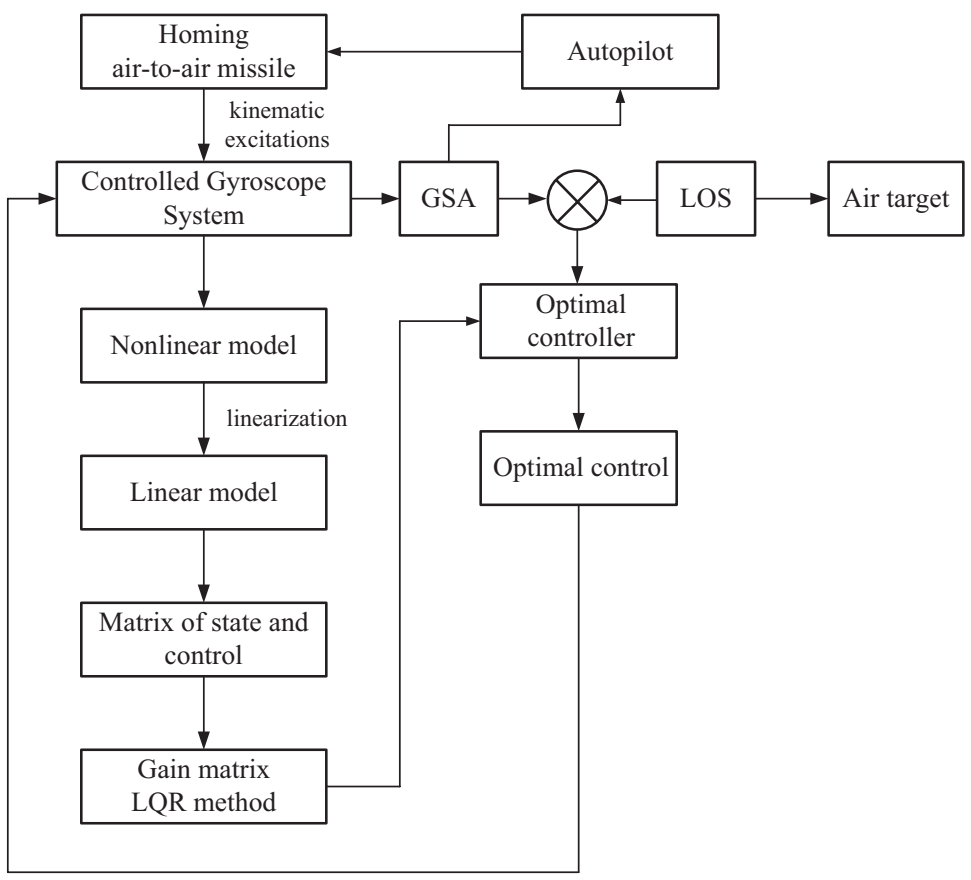

Figure 1. Block diagram of a gyroscope system control system operation within the homing process 


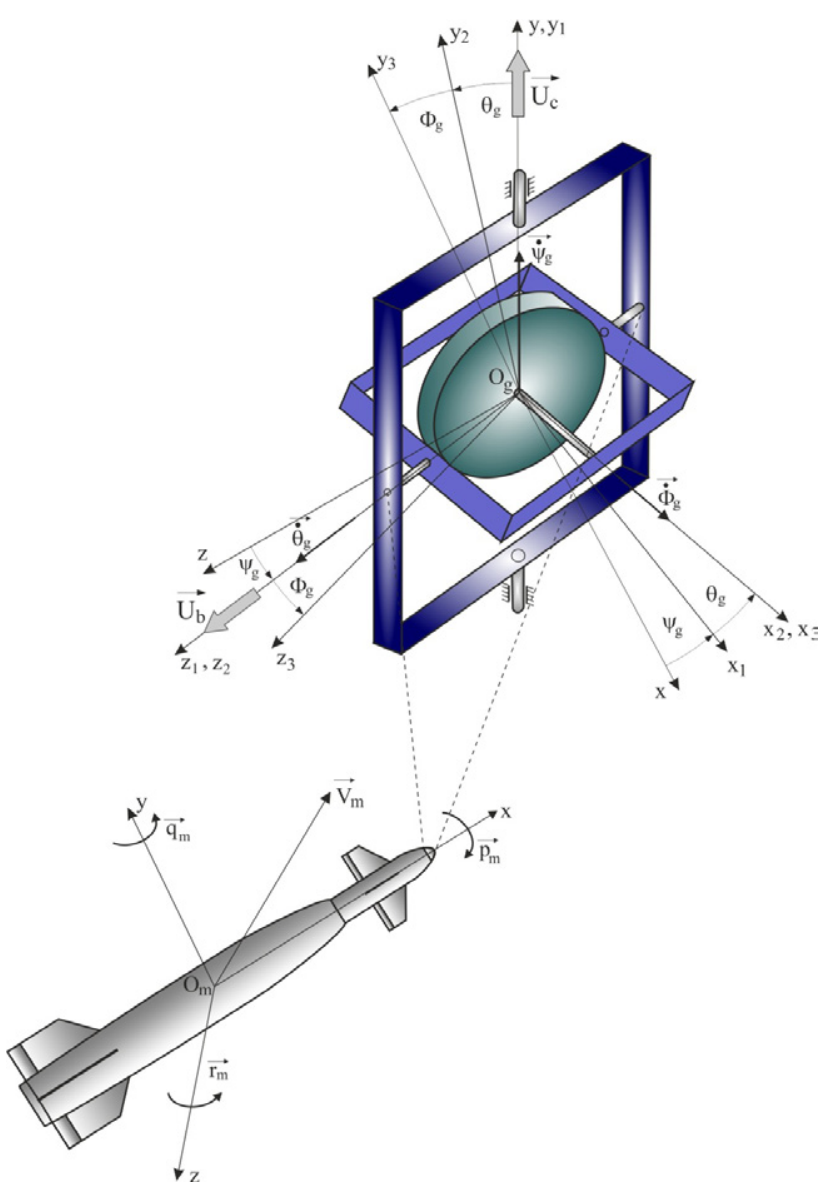

Figure 2. General view of a gyroscope system of a missile homing head

tors of force moments acting upon the inner and outer frame, respectively. Defining the coordinate systems, angular velocities and angles depicted in Figure 2 enable deriving the dynamic motion equations for the missile and gyroscope system that is reviewed later on in this paper.

A non-linear missile flight dynamics model has been derived using the Euler-Lagrange formalism. Equations describing the translational motion of a missile centre of gravity $O_{m}$ have been derived in a coordinate system associated with air streams (also called the speed or aerodynamic system) flowing around the missile and have the following form:

$$
\begin{aligned}
& m_{m} \frac{d V_{m}}{d t}=P \cos \left(\alpha_{m}\right) \cos \left(\beta_{m}\right)-m_{m} g \sin \left(\gamma_{m}\right)+F_{A_{x}} ; \quad \\
& m_{m} V_{m} \frac{d \gamma_{m}}{d t}=P \sin \left(\alpha_{m}\right)-m_{m} g \cos \left(\gamma_{m}\right)+F_{A_{y}}+Q_{y} ; \quad \\
& -m_{m} V_{m} \cos \left(\gamma_{m}\right) \frac{d \chi_{m}}{d t}=-P \cos \left(\alpha_{m}\right) \sin \left(\beta_{m}\right)+F_{A_{z}}+Q_{z},
\end{aligned}
$$

where: $V_{m}$ - missile velocity; $m_{m}-$ missile mass; $\chi_{m}-$ flight trajectory yaw angle; $\gamma_{m}$ - flight trajectory pitch angle; $\alpha_{m}$ - angle of attack; $\beta_{m}$ - angle of slip; $P$ - missile engine thrust; $g$ - gravitational acceleration; $F_{A x}, F_{A y}$,
$F_{A z}$ - aerodynamic action force; $Q_{y}$ - vertical plane control force; $Q_{z}$ - horizontal plane control force.

The equations describing the rotational motion of a missile around its centre of gravity $O_{m}$ have been derived in a coordinate system associated with the missile body, and have the following form:

$$
\begin{aligned}
& I_{m_{x}} \frac{d p_{m}}{d t}=\left(I_{m_{y}}-I_{m_{z}}\right) q_{m} r_{m}+M_{A_{x}} ; \\
& I_{m_{y}} \frac{d q_{m}}{d t}=\left(I_{m_{z}}-I_{m_{x}}\right) r_{m} p_{m}+M_{A_{y}}+M_{Q_{y}} ; \\
& I_{m_{z}} \frac{d r_{m}}{d t}=\left(I_{m_{x}}-I_{m_{y}}\right) q_{m} p_{m}+M_{A_{z}}+M_{Q_{z}},
\end{aligned}
$$

where: $I_{m_{x}}, I_{m_{y}}, I_{m_{z}}$ - missile moments of inertia relative to axis $x, y$ and $z$, respectively; $p_{m}, q_{m}, r_{m}$ - angular velocities of the missile - tilt, yaw and pitch velocity, respectively; $M_{A_{x}}, M_{A_{y}}, M_{A_{z}}$ - moments of aerodynamic forces; $M_{Q_{y}}, M_{Q_{z}}$ - moments of missile flight control forces.

The position of the missile and target relative to each other is determined by the following kinematic relationships (Krzysztofik \& Koruba, 2014):

$\frac{d \xi}{d t}=V_{t}\left[\cos \left(\gamma_{t}\right) \cos \left(\gamma_{L O S}\right) \cos \left(\chi_{L O S}-\chi_{t}\right)+\sin \left(\gamma_{t}\right) \sin \left(\gamma_{L O S}\right)\right]-$
$V_{m}\left[\cos \left(\gamma_{m}\right) \cos \left(\gamma_{L O S}\right) \cos \left(\chi_{L O S}-\chi_{m}\right)+\sin \left(\gamma_{m}\right) \sin \left(\gamma_{L O S}\right)\right]$,

$\xi \frac{d \gamma_{L O S}}{d t}=-V_{t}\left[\cos \left(\gamma_{t}\right) \sin \left(\gamma_{L O S}\right) \cos \left(\chi_{L O S}-\chi_{t}\right)-\sin \left(\gamma_{t}\right) \cos \left(\gamma_{L O S}\right)\right]+$ $V_{m}\left[\cos \left(\gamma_{m}\right) \sin \left(\gamma_{L O S}\right) \cos \left(\chi_{L O S}-\chi_{m}\right)-\sin \left(\gamma_{m}\right) \cos \left(\gamma_{L O S}\right)\right]$,

$-\xi \frac{d \chi_{L O S}}{d t} \cos \gamma_{L O S}=V_{t} \cos \left(\gamma_{t}\right) \sin \left(\chi_{L O S}-\chi_{t}\right)-$

$V_{m} \cos \left(\gamma_{m}\right) \sin \left(\chi_{L O S}-\chi_{m}\right)$,

where: $\xi$ - distance between the missile and target; $\gamma_{L O S}-$ LOS pitch angle; $\chi_{L O S}$ - LOS yaw angle; $V_{t}$ - target velocity; $\chi_{t}, \gamma_{t}$ - target velocity vector yaw and pitch angles.

Homing of a missile in on a target followed a proportional navigation algorithm:

$$
\dot{\gamma}_{m}=a_{1} \dot{\theta}_{g}, \dot{\chi}_{m}=a_{2} \dot{\psi}_{g},
$$

where: $a_{1}, a_{2}$ - guidance constants; $\dot{\theta}_{g}$ and $\dot{\psi}_{g}$ - angular velocities derived from the equations below.

Just like in the case of missile equations, the EulerLagrange was also used to derive a complete, non-linear motion dynamics model for a gyroscope system onboard a missile:

$$
\begin{aligned}
& \left(J_{g y_{2}}+J_{g y_{3}}\right) \frac{d \omega_{g y_{2}}}{d t}-m_{g} l_{g} \frac{d V_{g z_{2}}}{d t}-\left(J_{g z_{2}}+J_{g z_{3}}-J_{g x_{2}}\right) \omega_{g x_{2}} \omega_{g z_{2}}+ \\
& J_{g x_{3}} \omega_{g x_{3}} \omega_{g z_{2}}-m_{g} l_{g}\left[V_{g y_{2}} \omega_{g x_{2}}-V_{g x_{2}} V_{g y_{2}}\right]=U_{b}-U_{b f},
\end{aligned}
$$


$J_{g z_{1}} \frac{d \omega_{g z_{1}}}{d t}+\left(J_{g z_{2}}+J_{g z_{3}}\right) \frac{d}{d t}\left(\omega_{g z_{2}} \cos \theta_{g}\right)-J_{g x_{2}} \frac{d}{d t}\left(\omega_{g x_{2}} \sin \theta_{g}\right)-$

$J_{g x_{3}} \frac{d}{d t}\left(\omega_{g x_{3}} \sin \theta_{g}\right)+m_{g} l_{g} \frac{d}{d t}\left[V_{g y_{2}}\left(1+\cos \theta_{g}\right)\right]-$

$\left(J_{g x_{1}}-J_{g y_{1}}\right) \omega_{g x_{1}} \omega_{g y_{1}}-\left(J_{g z_{2}}+J_{g z_{3}}\right) \omega_{g z_{2}} \omega_{g y_{1}} \sin \theta_{g}+$

$\left(J_{g y_{2}}+J_{g y_{3}}\right) \omega_{g y_{2}} \omega_{g x_{1}}-\left(J_{g x_{2}} \omega_{g x_{2}}+J_{g x_{3}} \omega_{g x_{3}}\right) \omega_{g x_{1}} \cos \theta_{g}-$

$m_{g} V_{g x_{2}}\left[V_{g y_{1}} \cos \theta_{g}-l_{g} \omega_{g x_{1}} \sin \theta_{g}\right]-m_{g} V_{g y_{2}}\left[-V_{g x_{1}}+l_{g} \omega_{g y_{1}} \sin \theta_{g}\right]-$

$m_{g} V_{g z_{2}}\left[V_{g y_{1}} \sin \theta_{g}+l_{g} \omega_{g x_{1}}\left(1+\cos \theta_{g}\right)\right]=U_{c}-U_{c f}$,

where:

$$
\begin{aligned}
& \omega_{g x_{1}}=p_{m} \cos \psi_{g}+q_{m} \sin \psi_{g} ; \\
& \omega_{g y_{1}}=-p_{m} \sin \psi_{g}+q_{m} \cos \psi_{g} ; \\
& \omega_{g z_{1}}=\dot{\psi}_{g}+r_{m} ; \\
& \omega_{g x_{2}}=\omega_{g x_{1}} \cos \theta_{g}-\omega_{g z_{1}} \sin \theta_{g} ; \\
& \omega_{g y_{2}}=\omega_{g y_{1}}+\dot{\theta}_{g} ; \\
& \omega_{g z_{2}}=\omega_{g x_{1}} \sin \theta_{g}+\omega_{g z_{1}} \cos \theta_{g} ; \\
& \omega_{g x_{3}}=\omega_{g x_{2}}+n_{g} ; \\
& V_{g x_{1}}=V_{m} \cos \psi_{g} ; \\
& V_{g y_{1}}=-V_{m} \sin \psi_{g} ; \\
& V_{g x_{2}}=V_{g x_{1}} \cos \theta_{g}+l_{g} \omega_{g y_{1}} \sin \theta_{g} ; \\
& V_{g y_{2}}=V_{g x_{1}} \sin \theta_{g}-l_{g}\left(\omega_{g y_{1}} \sin \theta_{g}+\omega_{g y_{2}}\right)
\end{aligned}
$$

and $\psi_{g}, \theta_{g}, \Phi_{g}$ - angles defining the GS rotor spatial position; $J_{g x_{1}}, J_{g y_{1}}, J_{g z_{1}}-$ GS outer frame moments of inertia;
$J_{g x_{2}}, J_{g y_{2}}, J_{g z_{2}}-$ GS inner frame moments of inertia; $J_{g x_{3}}, J_{g y_{3}}, J_{g z_{3}}-$ GS rotor moment of inertia; $n_{g}-\mathrm{GS}$ rotor specific rotation speed; $m_{g}$ - system mass: inner frame - rotor; $l_{g}$ - distance of the rotor-inner frame system centre of gravity from the centre of rotation; $U_{b}, U_{c}-$ control moments; $U_{b f}, U_{c f}$ - moment of friction forces in the bearings of the inner and outer frames, respectively.

The research adopted viscous-type friction, therefore the moments $U_{b f}$ and $U_{c f}$ are determined by the relationships:

$$
U_{b f}=\eta_{b} \dot{\theta}_{g}, U_{c f}=\eta_{c} \dot{\psi}_{g},
$$

where: $\eta_{b}, \eta_{c}$ - attenuation factors in gyro frame suspension bearings.

A diagram covering the determination of optimal controls for a gyroscope system of an anti-aircraft air-air missile homing head is shown in Figure 3.

A linearized model of a controlled gyroscope system shall, therefore, be expressed in both vector and matrix forms:

$$
\frac{d \mathbf{x}_{\mathbf{g}}}{d t}=\mathbf{A}_{\mathbf{g}} \mathbf{x}_{\mathbf{g}}-\mathbf{B}_{\mathbf{g}} \mathbf{u}_{\mathbf{g}}
$$

where: $\mathbf{x}_{\mathbf{g}}$ - vector of state, $\mathbf{A}_{\mathbf{g}}$ - matrix of state, $\mathbf{B}_{\mathbf{g}}-$ control matrix, $\mathbf{u}_{\mathbf{g}}$ - control vector, with the following components:

$$
\mathbf{x}_{\mathbf{g}}=\left[\begin{array}{c}
\theta_{g} \\
\dot{\theta}_{g} \\
\psi_{g} \\
\dot{\psi}_{g}
\end{array}\right], \mathbf{A}_{\mathbf{g}}=\left[\begin{array}{cccc}
0 & 1 & 0 & 0 \\
0 & -\eta_{b} / J_{g k} & 0 & -J_{g o} n_{g} / J_{g k} \\
0 & 0 & 0 & 1 \\
0 & J_{g o} n_{g} / J_{g k} & 0 & -\eta_{c} / J_{g k}
\end{array}\right],
$$

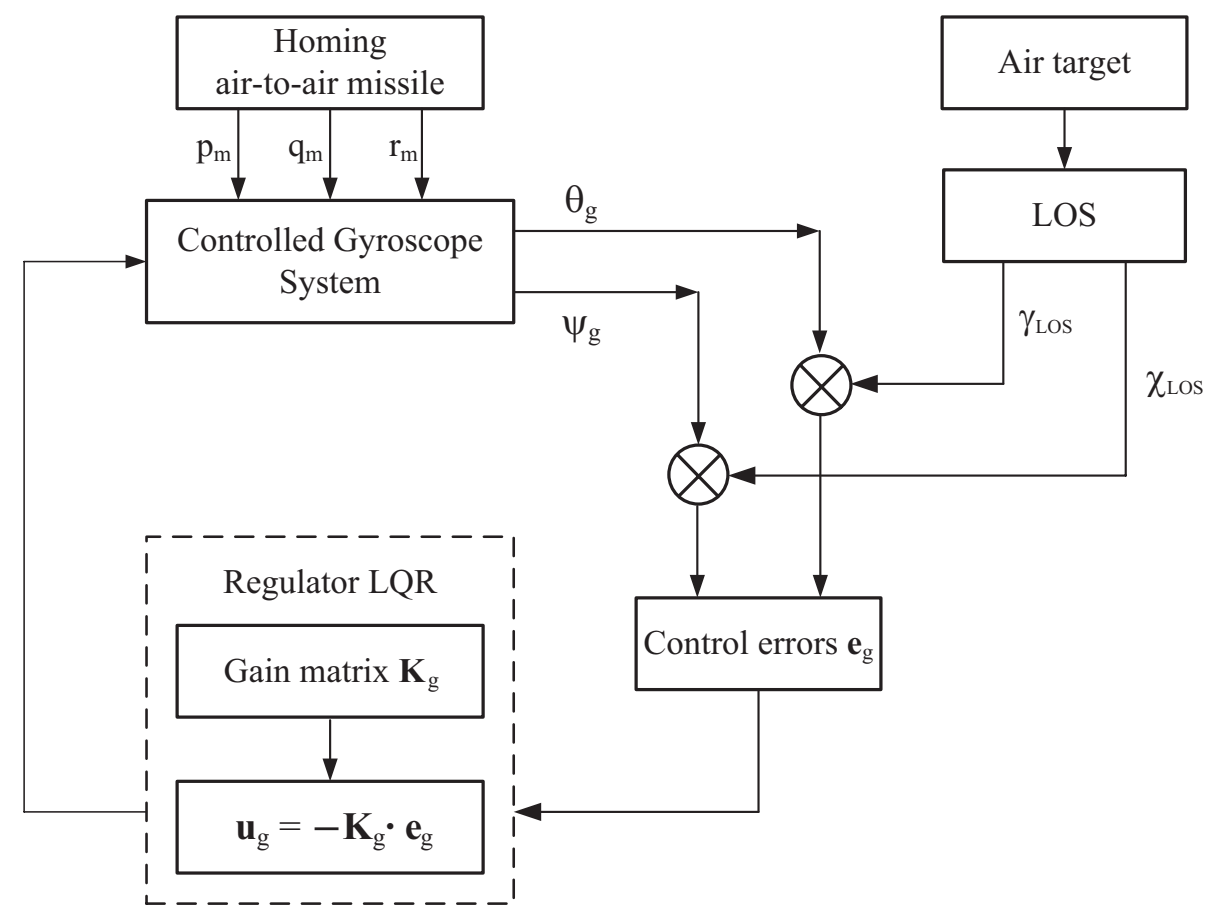

Figure 3. Diagram for the determination of optimal controls 


$$
\mathbf{B}_{\mathbf{g}}=\left[\begin{array}{cc}
0 & 0 \\
1 / J_{g k} & 0 \\
0 & 0 \\
0 & 1 / J_{g k}
\end{array}\right], \mathbf{u}_{\mathbf{g}}=\left[\begin{array}{l}
U_{b} \\
U_{c}
\end{array}\right],
$$

where: $J_{g o}, J_{g k}$ - moment of inertia of a GS rotor relative to the longitudinal and transverse axes, respectively.

Let a square quality indicator be known, with the following form (Lewis et al., 2012):

$$
J=\int_{0}^{\infty}\left(\mathbf{x}_{\mathbf{g}}^{\mathrm{T}} \mathbf{Q}_{\mathbf{g}} \mathbf{x}_{\mathbf{g}}+\mathbf{u}_{\mathbf{g}}^{\mathrm{T}} \mathbf{R}_{\mathbf{g}} \mathbf{u}_{\mathbf{g}}\right) d t,
$$

where: $\mathbf{Q}_{\mathbf{g}}, \mathbf{R}_{\mathbf{g}}$ - weight matrices selected via experiments.

The control law shall be expressed as follows:

$$
\mathbf{u}_{\mathrm{g}}=-\mathbf{K}_{\mathrm{g}} \mathbf{e}_{\mathrm{g}} \text {, }
$$

where: $\mathbf{e}_{\mathbf{g}}=\left(\mathbf{x}_{\mathbf{g}}-\mathbf{x}_{\mathbf{g d}}\right)-$ control errors;

$\mathbf{x}_{\mathbf{g d}}=\left[\begin{array}{llll}\gamma_{L O S} & \dot{\gamma}_{L O S} & \chi_{L O S} & \dot{\chi}_{L O S}\end{array}\right]^{T}-$ vector of desired state variables, with the components derived from Equations (7)-(9).

The feedback matrix $\mathbf{K}_{\mathbf{g}}$ is determined based on the following relationship:

$$
\mathbf{K}_{\mathrm{g}}=\mathbf{R}_{\mathrm{g}}^{-1} \mathbf{B}_{\mathrm{g}}^{\mathrm{T}} \mathbf{P}_{\mathbf{g}} .
$$

For the purposes of the paper, the matrix $\mathbf{K}_{\mathbf{g}}$ has been determined using the lqr (Tewari, 2002) Matlab function:

$$
\mathbf{K}_{\mathbf{g}}=\operatorname{lqr}\left(\mathbf{A}_{\mathbf{g}}, \mathbf{B}_{\mathbf{g}}, \mathbf{Q}_{\mathrm{g}}, \mathbf{R}_{\mathbf{g}}\right) \text {. }
$$

The matrix $\mathbf{P}_{\mathbf{g}}$ present in the equation is derived from the algebraic Riccati equation:

$$
\mathbf{A}_{\mathrm{g}}^{\mathrm{T}} \mathbf{P}_{\mathrm{g}}+\mathbf{P}_{\mathrm{g}} \mathbf{A}_{\mathrm{g}}-\mathbf{P}_{\mathrm{g}} \mathbf{B}_{\mathrm{g}} \mathbf{R}_{\mathrm{g}}^{-1} \mathbf{B}_{\mathrm{g}}^{\mathrm{T}} \mathbf{P}_{\mathrm{g}}+\mathbf{Q}_{\mathrm{g}}=0
$$

\section{Numerical simulation results}

This section presents the results of simulation tests conducted in order to evaluate the LQR designed for controlling a gyroscope system during the process of homing an air-air missile in on a manoeuvring air target. The numerical simulations were performed using the Matlab/ Simulink software, utilizing the procedure ode45, with a variable integration step (Baranowski, 2013), for the following parameters:

Target and missile initial parameters

$X_{t}=1500 \mathrm{~m} ; Y_{t}=500 \mathrm{~m} ; Z_{t}=350 \mathrm{~m} ; V_{t}=200 \mathrm{~m} / \mathrm{s}$;

$\gamma_{t}=0.0 \mathrm{rad} ; \chi_{t}=0.0 \mathrm{rad} ; \Omega_{t}=0.15 \mathrm{rad} / \mathrm{s}$;

$X_{m}=1000 \mathrm{~m} ; Y_{m}=500 \mathrm{~m} ; Z_{m}=100 \mathrm{~m} ; V_{m}=50 \mathrm{~m} / \mathrm{s}$.

Guidance constants

$a_{1}=a_{2}=3.5$.

Gyroscope system parameters

$J_{g o}=5 \cdot 10^{-4} \mathrm{kgm}^{2} ; J_{g k}=2.5 \cdot 10^{-4} \mathrm{kgm}^{2} ;$

$n_{g}=600 \mathrm{rad} / \mathrm{s} ; \eta_{b}=\eta_{c}=0.01 \mathrm{Nm} / \mathrm{s}$.
LQR parameters (weight matrices)

$$
\mathbf{Q}_{\mathbf{g}}=\left[\begin{array}{cccc}
200 & 0 & 0 & 0 \\
0 & 100 & 0 & 0 \\
0 & 0 & 10 & 0 \\
0 & 0 & 0 & 100
\end{array}\right] ; \mathbf{R}_{\mathrm{g}}=\left[\begin{array}{ll}
5 & 0 \\
0 & 5
\end{array}\right]
$$

The operation of an LQR was compared with the operation of a PD regulator, the coefficients of which have been selected optimally so as not to exceed permissible control values during the manoeuvring and to obtain a lower target function value. This was the reason why the study utilized the optimization algorithm reviewed by Awrejcewicz and Koruba (2012):

$$
\begin{aligned}
& U_{b}=-k_{b}\left(\theta_{g}-\gamma_{L O S}\right)+k_{c}\left(\psi_{g}-\chi_{L O S}\right)-h_{g}\left(\dot{\theta}_{g}-\dot{\gamma}_{L O S}\right), \\
& U_{c}=-k_{c}\left(\theta_{g}-\gamma_{L O S}\right)-k_{b}\left(\psi_{g}-\chi_{L O S}\right)-h_{g}\left(\dot{\psi}_{g}-\dot{\chi}_{L O S}\right), \\
& k_{b}=2.5 ; k_{c}=3.46 ; h_{g}=1.73 .
\end{aligned}
$$

Figures 4-13 show selected simulation test results. Figure 4 reveals a missile intercepting a manoeuvring air target after $5.3 \mathrm{~s}$. Figures 5 and 6 demonstrate good convergence of the desired and executed flight angles. In the case of using an LQR for controlling a GS, fast and effective overlapping of the gyroscope axis and the target line-of-sight can also be noticed - Figures 7, 10 and 11. There are only slight deviations of the gyroscope system axis path from the set value in the initial phase of homing a missile on a target (Figure 8).

The operational efficiency of an LQR was also assessed for a scenario in which a missile is impacted by highamplitude harmonic kinematic forces over a short time interval. These forces, converging through the gyroscope frame bearings, adversely impact the stabilization of a GS axis along the target LOS. In Figures 10 and 11, it is seen that the control system, together with the LQR regulator, rapidly restores the GS axis to a desired position. This is also confirmed by the results in Figure 12. After the disturbance passes, the actual and set gyroscope axis path fully coincide.

The presented research results indicate that optimal regulator parameters enable the constant up-keep of a stable target in the line of sight of the homing head optical system (Figures 7a, 10a and 11a). This can be of significant

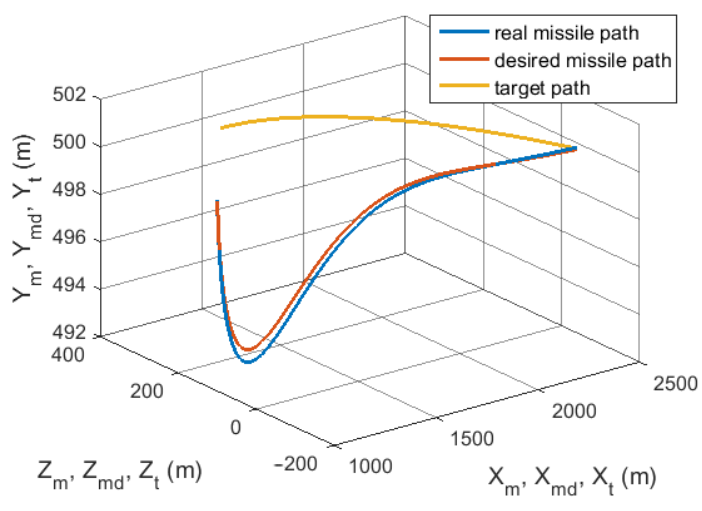

Figure 4. Missile and target flight trajectories 


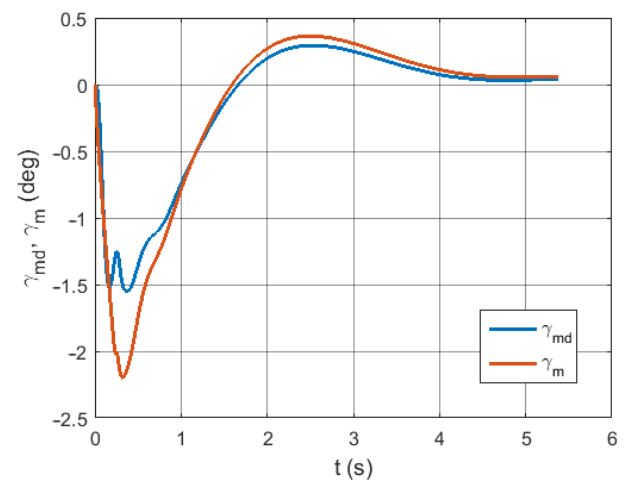

a)

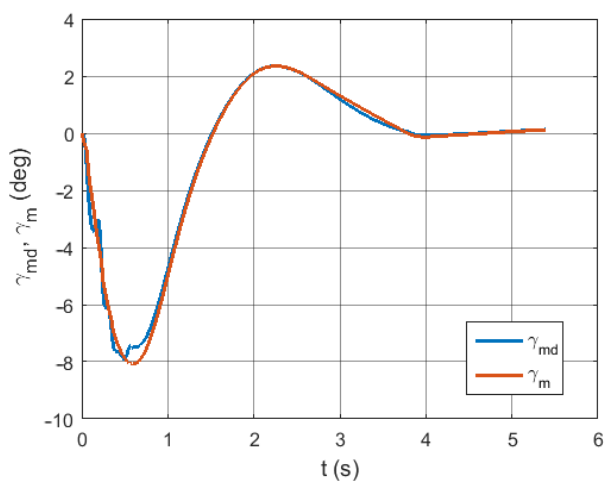

b)

Figure 5. Actual $\gamma_{m}$ and desired $\gamma_{m d}$ missile flight angles over time, for: a) LQR, b) PD regulator

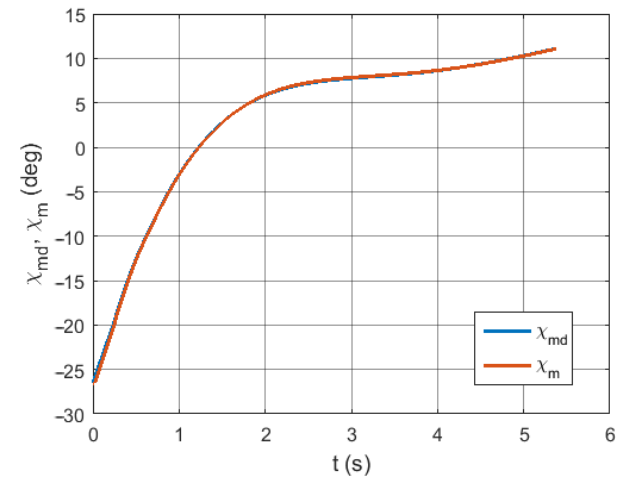

a)

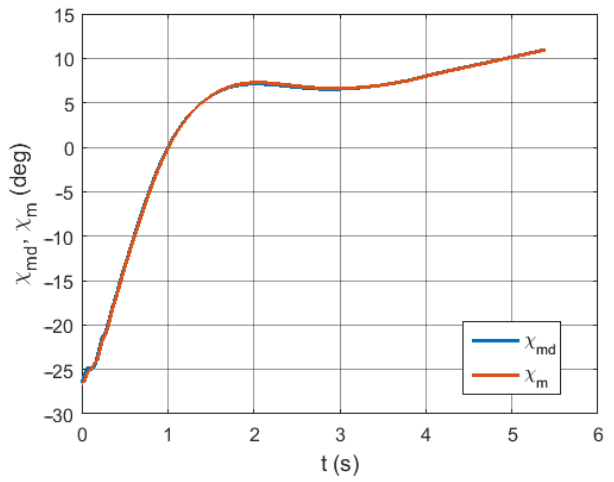

b)

Figure 6. Actual $\chi_{m}$ and desired $\chi_{m d}$ missile flight angles over time, for: a) LQR, b) PD regulator

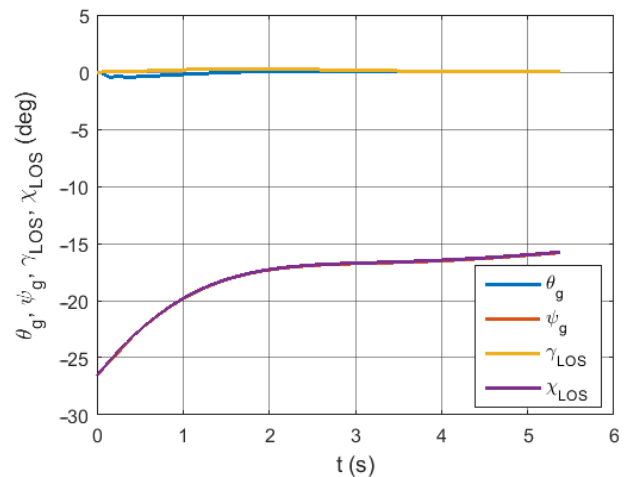

a)

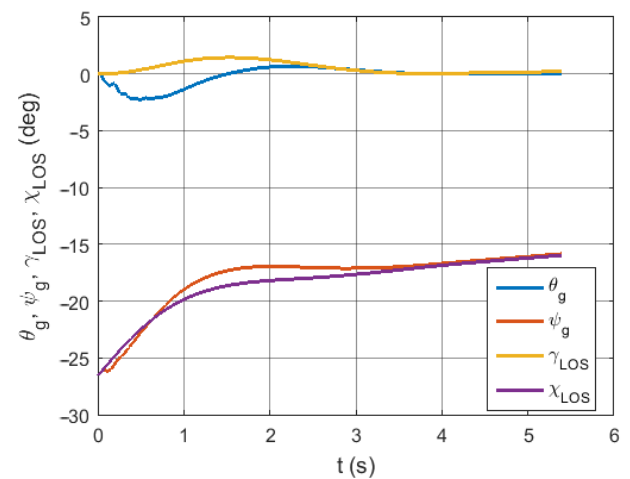

b)

Figure 7. Pitch and yaw angles for LOS and gyroscope system axis over time, for: a) LQR, b) PD regulator

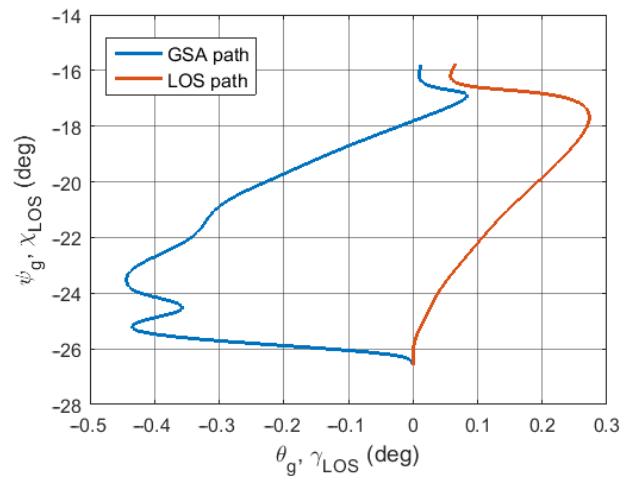

a)

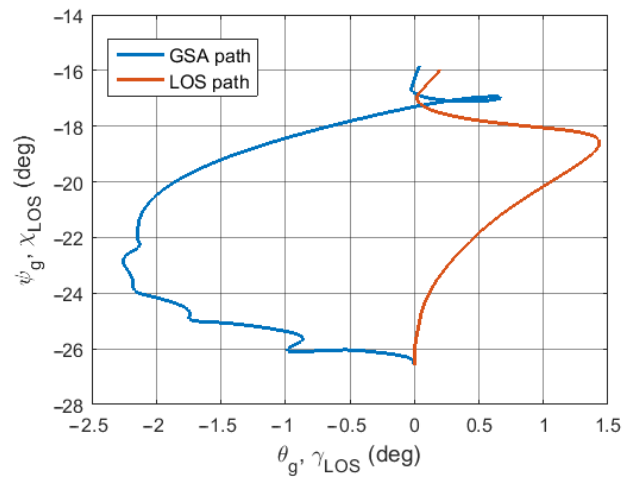

b)

Figure 8. Actual and desired gyroscope system trajectories, for: a) LQR, b) PD regulator 


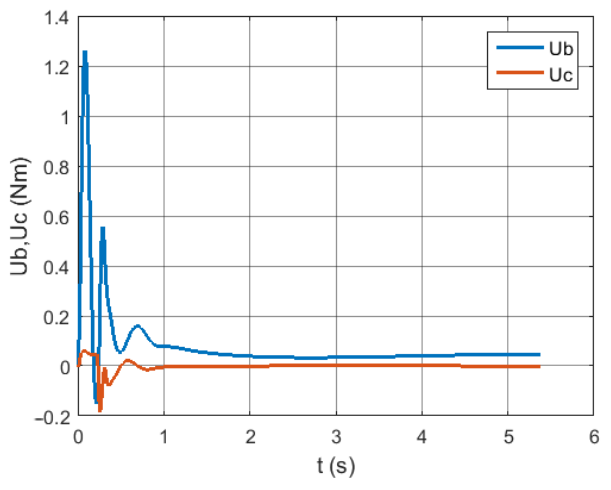

a)

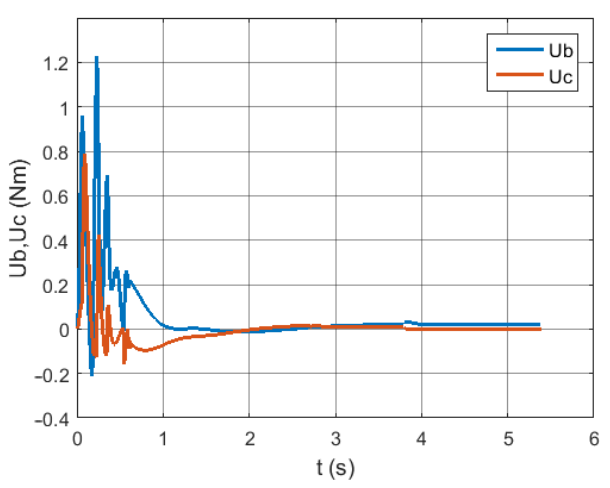

b)

Figure 9. Control moments over time, for: a) LQR, b) PD regulator

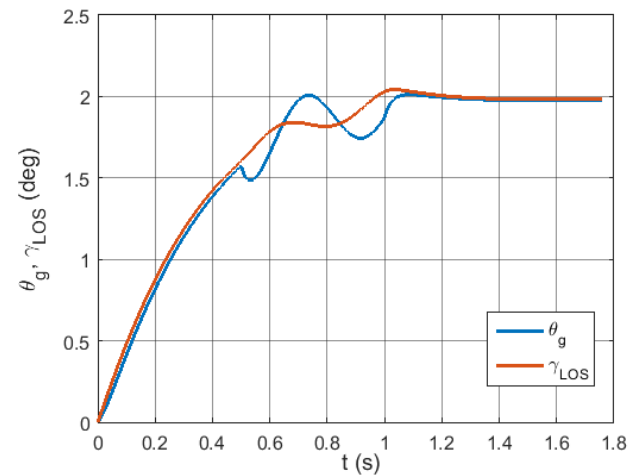

a)

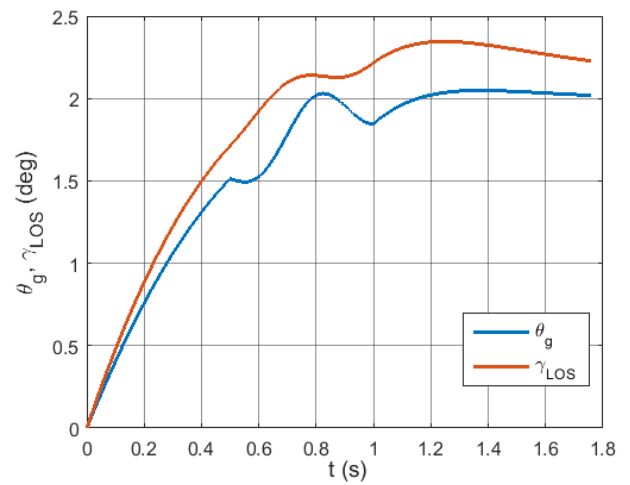

b)

Figure 10. Pitch angle for LOS and gyroscope system axis, for: a) LQR, b) PD regulator

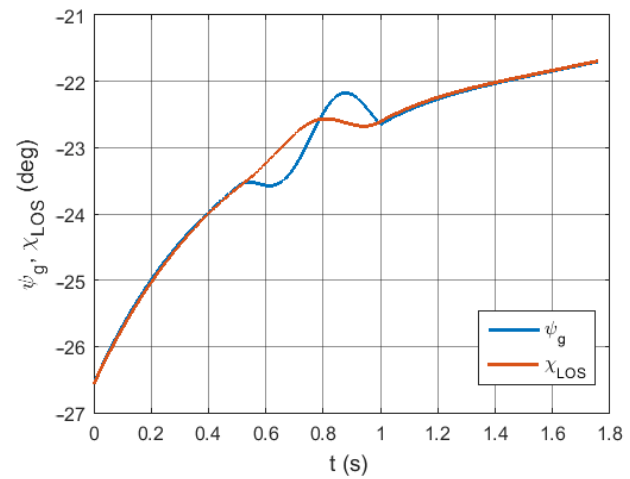

a)

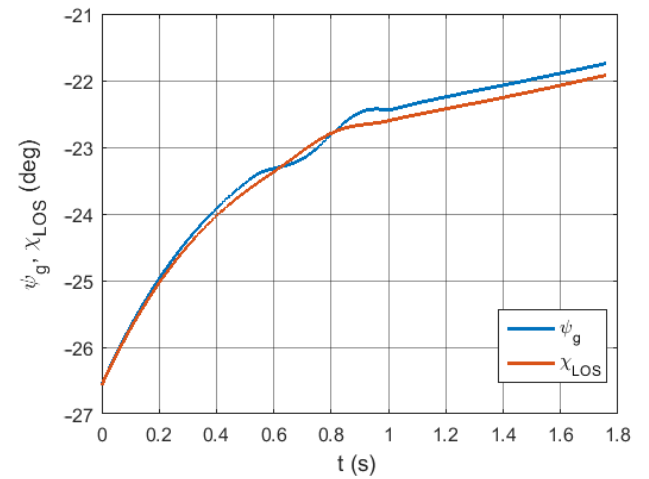

b)

Figure 11. Yaw angle for LOS and gyroscope system axis, for: a) LQR, b) PD regulator

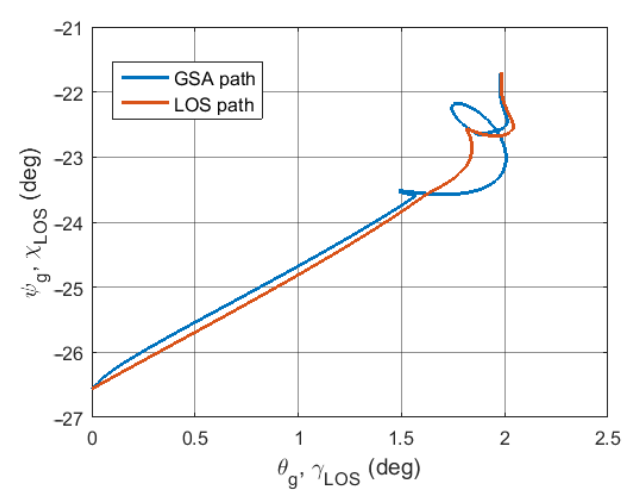

a)

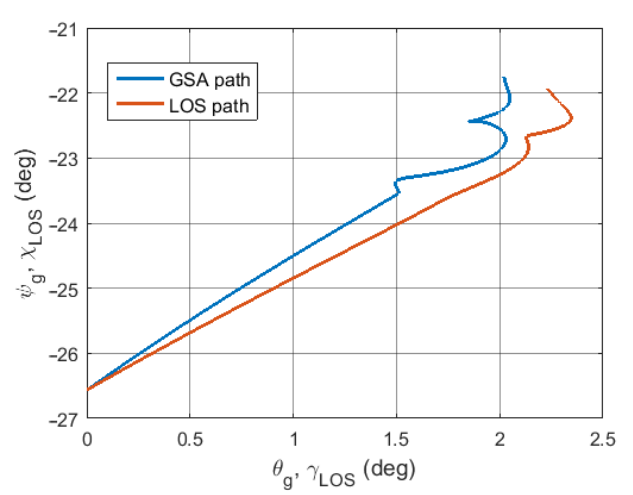

b)

Figure 12. Actual and desired gyroscope system axis trajectories, for: a) LQR, b) PD regulator 


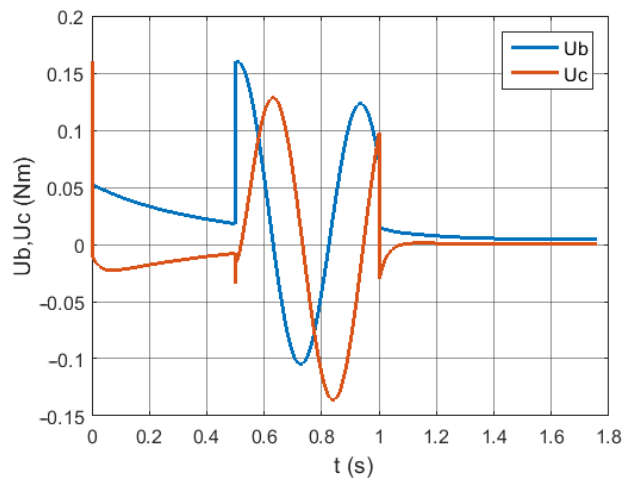

a)

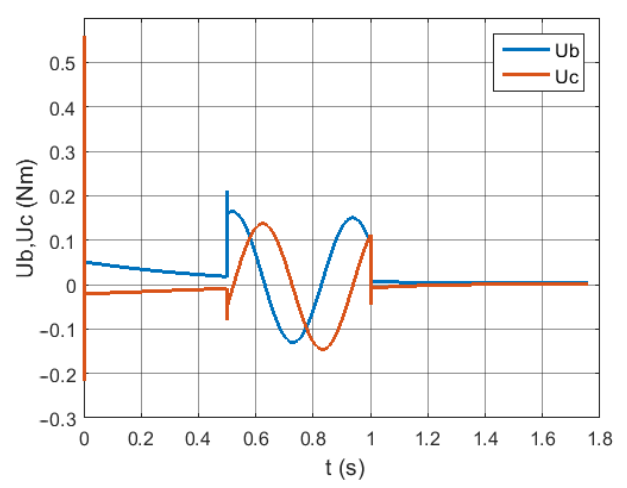

b)

Figure 13. Control moments over time, for: a) LQR, b) PD regulator

importance when a target is detected with high angular deflection with respect to the missile's longitudinal axis relative to the target line of sight.

It should be stressed that the control moments adopt minor values (Figures 9a and 13a) that determine their feasibility in the conditions of an actual homing process. The operation of an optimal regulator for a gyroscope system is also stable under the impact of large interference by the missile deck (Figure 12a).

\section{Disclosure statement}

The authors do not have any competing financial, professional, or personal interests from other parties.

\section{Conclusions}

The obtained tests results indicate the correctness of the original operation developed by the authors of the control algorithm for a gyroscope in a homing missile. The outcome of the tests presented in the research paper also demonstrate the high efficiency of the optimal control algorithm in controlling a gyroscope system within the process of homing an air to air missile on a manoeuvring air target. Furthermore, the conducted comparative analysis showed that an LQR ensured higher homing precisions of a missile on a manoeuvring air target, relative to a PD regulator, despite the fact that the parameters of the latter were selected in an optimal manner, following the Golubencev method. This can be clearly seen in the conditions of active strong external interference. This is of significant importance in terms of the homing process, since it determines in many cases effective anti-aircraft defence.

The future research of the authors will focus on including the non-stationarity of a guidance system (time-variable state matrix components) and the implementation of a developed controlled algorithm within an actual homing missile, as well as its verification in field conditions.

\section{References}

Awrejcewicz, J., \& Koruba, Z. (2012). Classical mechanics: applied mechanics and mechatronics. Springer. https://doi.org/10.1007/978-1-4614-3978-3

Balakrishnan, S. N., Tsourdos, A., \& White, B. A. (2013). Advances in missile guidance, control, and estimation. CRC Press.

Baranowski, L. (2013). Effect of the mathematical model and integration step on the accuracy of the results of computation of artillery projectile flight parameters. Bulletin of the Polish Academy of Sciences. Technical Sciences, 61(2), 475-484. https://doi.org/10.2478/bpasts-2013-0047

Chen, H.-K., \& Ge, Z.-M. (2005). Bifurcations and chaos of a two-degree-of-freedom dissipative gyroscope. Chaos, Solitons and Fractals, 24(1), 125-136.

https://doi.org/10.1016/j.chaos.2004.07.028

Chen, S.-C., Kuo, C.-L., Lin, C.-H., Hsu, C.-H., \& Tsui, C.-K. (2013). Applications of fuzzy sliding mode control for a gyroscope system. Abstract and Applied Analysis, 2013, Article ID 931285. https://doi.org/10.1155/2013/931285

Gapinski, D., \& Krzysztofik, I. (2014). The process of tracking an air target by the designed scanning and tracking seeker. In Proceedings of the 15th International Carpathian Control Conference, IEEE (pp. 129-134). Velke Karlovice, Czech Republic. https://doi.org/10.1109/CarpathianCC.2014.6843583

Gapiński, D., Krzysztofik, I., \& Koruba, Z. (2018). Multi-channel, passive, short-range anti-aircraft defence system. Mechanical Systems and Signal Processing, 98, 802-815. https://doi.org/10.1016/j.ymssp.2017.05.032

Gapiński, D., \& Stefański, K. (2014). A control of modified optical scanning and tracking head to detection and tracking air targets. Solid State Phenomena, 210, 145-155. https://doi.org/10.4028/www.scientific.net/SSP.210.145

Ge, Z.-M., \& Lee, J.-K. (2005). Chaos synchronization and parameter identification for gyroscope system. Applied Mathematics and Computation, 163(2), 667-682. https://doi.org/10.1016/j.amc.2004.04.008

Grzyb, M., \& Stefański, K. (2016). The use of special algorithm to control the flight of anti-aircraft missile. In Proceedings of the 22th International Conference Engineering Mechanics (pp. 174-177). Institute of Thermomechanics, Academy of Sciences of the Czech Republic, Svratka, Czech Republic.

Koruba, Z., \& Krzysztofik, I. (2013). An algorithm for selecting optimal controls to determine the estimators of the coefficients of a mathematical model for the dynamics of a self-propelled 
anti-aircraft missile system. In Proceedings of the Institution of Mechanical Engineers, Part K: Journal of Multi-body Dynamics, 227(1), 12-16. https://doi.org/10.1177/1464419312455967

Krzysztofik, I., \& Koruba, Z. (2014). Mathematical model of movement of the observation and tracking head of an unmanned aerial vehicle performing ground target search and tracking. Journal of Applied Mathematics, 2014, Article ID 934250. https://doi.org/10.1155/2014/934250

Krzysztofik, I., Takosoglu, J., \& Koruba Z. (2017). Selected methods of control of the scanning and tracking gyroscope system mounted on a combat vehicle. Annual Reviews in Control, 44, 173-182. https://doi.org/10.1016/j.arcontrol.2016.10.003

Lei, Y., Xu, W., \& Zheng, H. (2005). Synchronization of two chaotic nonlinear gyros using active control. Physics Letters A, 343(13), 153-158. https://doi.org/10.1016/j.physleta.2005.06.020

Lewis, F. L., Vrabie, D. L., \& Syrmos, V. L. (2012). Optimal control (3rd ed.). John Wiley \& Sons. https://doi.org/10.1002/9781118122631

Polo, M. P., Albertos, P., \& Galiano, J. A. B. (2008). Tuning of a PID controlled gyro by using the bifurcation theory. Systems \& Control Letters, 57(1), 10-17. https://doi.org/10.1016/j.sysconle.2007.06.007

Roopaei, M., Jahromi, M. Z., John, R., \& Lin, T-C. (2010). Unknown nonlinear chaotic gyros synchronization using adaptive fuzzy sliding mode control with unknown dead-zone input. Communications in Nonlinear Science and Numerical Simulation, 15(9), 2536-2545.

https://doi.org/10.1016/j.cnsns.2009.09.022

Sargolzaei, M., Yaghoobi, M., \& Yazdi, R. (2013). Modeling and synchronization of chaotic gyroscopes using TS fuzzy approach. Advance in Electronic and Electric Engineering, 3(3), 339-346.

Tewari, A. (2002). Modern control design with MATLAB and SIMULINK. John Wiley \& Sons.

Wang, C-C., \& Yau, H-T. (2011). Nonlinear dynamic analysis and sliding mode control for a gyroscope system. Nonlinear Dynamics, 66, 53-65. https://doi.org/10.1007/s11071-010-9910-4

Yau, H-T. (2008). Chaos synchronization of two uncertain chaotic nonlinear gyros using fuzzy sliding mode control. $\mathrm{Me}$ chanical Systems and Signal Processing, 22(2), 408-418. https://doi.org/10.1016/j.ymssp.2007.08.007

Zarchan, P. (2012). Tactical and strategic missile guidance (6th ed.). AiAA Inc. https://doi.org/10.2514/4.868948

\section{Notations}

Variables and functions

$X_{t}, Y_{t}, Z_{t}$ - target position coordinates;

$X_{m}, Y_{m}, Z_{m}$ - missile position coordinates;

$X_{m d}, Y_{m d}, Z_{m d}$ - desired position coordinates of missile;

$\gamma_{m}, \gamma_{m d}-$ actual and desired pitch angles of missile velocity vector, respectively;

$\chi_{m}, \chi_{m d}-$ actual and desired yaw angles of missile velocity vector, respectively;

$\gamma_{L O S}-$ LOS pitch angle;

$\chi_{L O S}$ - LOS yaw angle;

$\psi_{g}$ - the rotation angle of the outer frame of the gyroscope system;

$\theta_{g}$ - the rotation angle of the inner frame of the gyroscope system;

$U_{b}, U_{c}$ - control moments.

\section{Abbreviations}

GSA - Gyroscope System Axis;

GS - Gyroscope System;

LOS - Line-of-Sight;

PID - Proportional-Integral-Derivative;

PD - Proportional-Derivative;

LQR - Linear-Quadratic Regulator. 